\title{
Quantum phase transitions across a $p$-wave Feshbach resonance
}

\author{
V. Gurarie, L. Radzihovsky, and A. V. Andreev \\ Department of Physics, University of Colorado, Boulder CO 80309
}

(Dated: August 31, 2018)

\begin{abstract}
We study a single-species polarized Fermi gas tuned across a narrow $p$-wave Feshbach resonance. We show that in the course of a BEC-BCS crossover the system can undergo a magnetic fieldtuned quantum phase transition from a $p_{x}$-wave to a $p_{x}+i p_{y}$-wave superfluid. The latter state, that spontaneously breaks time-reversal symmetry, furthermore undergoes a topological $p_{x}+i p_{y}$ to $p_{x}+i p_{y}$ transition at zero chemical potential $\mu$. In two-dimensions, for $\mu>0$ it is characterized by a Pfaffian ground state exhibiting topological order and non-Abelian excitations familiar from fractional quantum Hall systems.
\end{abstract}

PACS numbers: 03.75.Ss, 67.90.+z, 74.20.Rp

Recent progress in controlling atomic interactions via an s-wave Feshbach resonance (FR) has led to a realization of an s-wave paired fermionic superfluid (SF) in a variety of degenerate atomic gases[1-3]. By tuning through FR such paired superfluid has been studied along the crossover between the BCS regime of weakly-paired, strongly overlapping Cooper pairs, and the BEC regime of tightly bound, weakly-interacting diatomic molecules.

Although the study of this crossover has received considerable attention $[4,5]$, its equilibrium properties are already qualitatively well described by early seminal works $[6,7]$. Furthermore, for a narrow FR, the thermodynamics can even be accurately computed analytically across the full range of the BCS-BEC crossover, with corrections controlled by the ratio of the FR width to Fermi energy $[8]$.

In contrast, superfluids paired at a finite angularmomentum are characterized by richer order parameters (as exemplified by a $p$-wave paired ${ }^{3} \mathrm{He}$, heavyfermion compounds, and $d$-wave high- $\mathrm{T}_{c}$ superconductors), and therefore admit phase transitions within the $\mathrm{SF}$ phase. Recent observation of a $p$-wave FR $[9,10]$ in ${ }^{40} \mathrm{~K}$ and ${ }^{6} \mathrm{Li}$, prepared in identical spin states, demonstrates a promising mechanism for a realization of $p$-wave superfluidity, attracting recent theoretical attention [1113]. Because Pauli exclusion principle prevents identical fermionic atoms from scattering via $s$-wave channel, such $p$-wave scattering dominates. The tuning of the atomic interaction through such FR should allow access to aforementioned phase transitions and provides strong motivation for our study.

In this Letter, we study a $p$-wave superfluidity in a single-species polarized Fermi gas tuned across a $p$-wave FR. We show analytically that at low $T$, in the course of a BEC-BCS crossover this system can exhibit a secondorder quantum phase transition as a function of FR detuning $\omega_{0}$ (energy of the quasi-bound molecular state) from a $p_{x}$-wave to a time-reversal breaking $p_{x}+i p_{y^{-}}$ wave superfluid. In three dimensions (3D), for a positive chemical potential $\mu>0$, the $p_{x}+i p_{y}$-wave superfluid exhibits gapless single particle excitations with momen- tum $\mathbf{p}= \pm p_{F} \hat{\mathbf{z}}$, that become gapped for $\mu<0$. Consequently, on quite general grounds we predict that in addition to above $p_{x}$ to $p_{x}+i p_{y}$ phase transition, the latter SF must furthermore undergo a topological quantum phase transition at $\omega_{0}(\mu=0)$, from a gapped to gapless $p_{x}+i p_{y}$ SF state[14] (Fig. 1(a,b)) Associated thermodynamic changes (e.g., a heat capacity changing from activated to power-law form) should be observable.
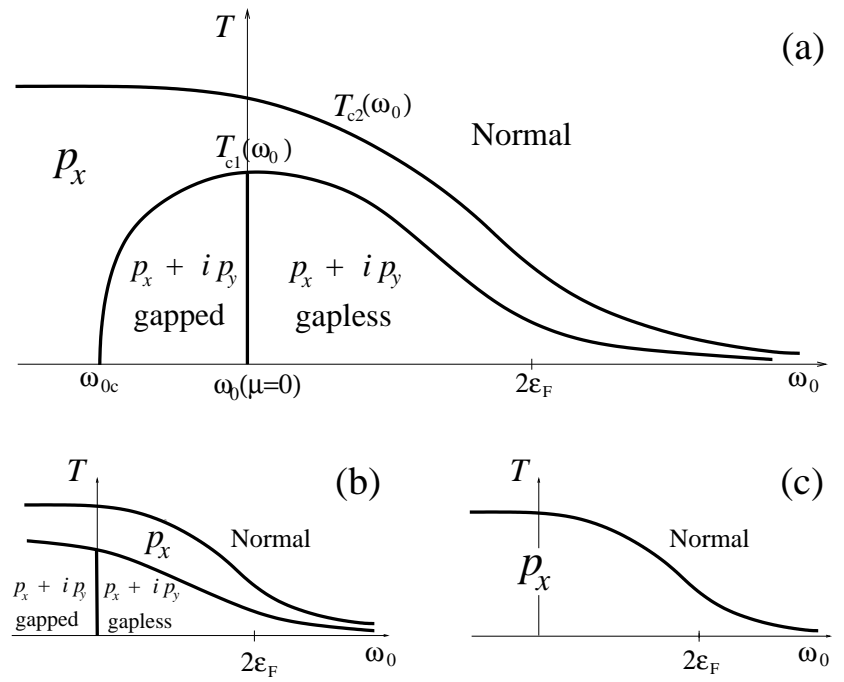

FIG. 1: (a) A schematic temperature-detuning phase diagram near a $p$-wave $\mathrm{FR}$, for intermediate splitting of $m=0, \pm 1 \mathrm{FR}$ (see below). It shows a 2 nd-order $p_{x}$ to $p_{x}+i p_{y} \mathrm{SF}$ transition at $\omega_{0 c}$ as well as a topological phase transition at $\omega_{0}(\mu=0)$ between two different $p_{x}+i p_{y}$ phases. (b), (c) show alternative phase diagrams for smaller and larger values of FR splitting, respectively.

Moreover, when confined to 2D, for $\mu>0$, the $p_{x}+i p_{y}$ superfluid is described by a fully-gapped Pfaffian (MooreRead) ground state [15], that exhibits a subtle topological order characterized by excitations with non-Abelian statistics, familiar from fractional quantum Hall systems. At $\omega_{0}(\mu=0)$, this fully-gapped superfluid has been demonstrated to undergo a topological quantum phase transition to another strongly-coupled gapped $p_{x}+i p_{y^{-}}$ wave superfluid $[16,17]$. 
The accuracy of our theory is controlled by the ratio of the width of the FR (molecular decay rate) to $\epsilon_{F}$. Because the low-energy decay rate of a finite angular momentum diatomic molecule is suppressed by the centrifugal barrier, our theory can be made arbitrarily accurate by reducing the atom density, $n$.

We start with a Hamiltonian of spinless fermionic atoms, resonantly interacting through a diatomic molecular state with internal angular momentum ("spin") $\ell=1$ :

$$
\begin{aligned}
H & =\sum_{p} \epsilon_{p} \hat{a}_{\mathbf{p}}^{\dagger} \hat{a}_{\mathbf{p}}+\sum_{\mathbf{p}, \alpha}\left(\epsilon_{\alpha}+\frac{\epsilon_{p}}{2}\right) \hat{b}_{\alpha \mathbf{p}}^{\dagger} \hat{b}_{\alpha \mathbf{p}} \\
& +\sum_{\mathbf{p}, \mathbf{q}, \alpha} \frac{g_{p}}{\sqrt{V}} p_{\alpha}\left(\hat{b}_{\alpha \mathbf{q}} \hat{a}_{\mathbf{p}+\frac{\mathbf{q}}{2}}^{\dagger} \hat{a}_{-\mathbf{p}+\frac{\mathbf{q}}{2}}^{\dagger}+h . c .\right) .
\end{aligned}
$$

Here $\hat{a}_{\mathbf{p}}^{\dagger}$ is the creation operator of an atom with momentum $\mathbf{p}$ and kinetic energy $\epsilon_{p}=p^{2} / 2 m$. $\hat{b}_{\alpha \mathbf{q}}^{\dagger}$ creates a molecule with momentum q, kinetic energy $\epsilon_{q} / 2$, and $\alpha$ labels its spin polarization. $V$ is the volume of the system. $\epsilon_{\alpha}$ controls the molecular rest energy related to FR detuning, that can in general be polarization dependent[9]. The fermion-molecule matrix element $g_{p}$ is a constant $g_{0}$ for $p \ll \Lambda$, quickly dropping off to zero for $p \gg \Lambda$. The ultraviolet (UV) cutoff $\Lambda$ is associated with the smallest length scale in the problem, the size of the molecular (closed-channel) bound state.

We first study elastic resonant scattering of two atoms with momenta $\mathbf{p}$ and $-\mathbf{p}$ into states $\mathbf{p}^{\prime}$ and $-\mathbf{p}^{\prime}$. This will enable us to relate the bare parameters $\epsilon_{\alpha}$ appearing in $H$ to the experimental FR detuning $\omega_{\alpha}$. In vacuum this process can only proceed through an intermediate molecular bound state, created by $\hat{b}_{\alpha 0}^{\dagger}$, at zero momentum and energy $E=p^{2} / m$. The scattering amplitude $f\left(\mathbf{p}, \mathbf{p}^{\prime}\right)$ is determined by the 4 -fermion amputated correlation function. It can be deduced from the molecular Green's function at zero momentum, $D_{\alpha \beta}(E)=$ $\delta_{\alpha \beta}\left[E-\epsilon_{\alpha}-\Pi(E)\right]^{-1}$, expressed in terms of the molecular self-energy (fermionic polarization "bubble") $\Pi(E)$, given by $\Pi(E)=\int \frac{d \omega}{2 \pi} \frac{d^{3} k}{(2 \pi)^{3}} \frac{\frac{2}{3} i g_{k}^{2} k^{2}}{\left(E-\omega-\frac{k^{2}}{2 m}+i 0\right)\left(\omega-\frac{k^{2}}{2 m}+i 0\right)}$. Evaluating the integrals (with $\epsilon_{\Lambda} \gg E$ ), we arrive at an exact expression for the $p$-wave scattering amplitude

$$
f\left(\mathbf{p}, \mathbf{p}^{\prime}\right)=\sum_{\alpha} \frac{-\left(m g_{0}^{2} / 2 \pi\right) p_{\alpha} p_{\alpha}^{\prime}}{\frac{p^{2}}{m}\left(1+c_{2}\right)-\epsilon_{\alpha}+c_{1}+i \frac{m g_{0}^{2}}{6 \pi} p^{3}},
$$

with $c_{1}=\frac{m}{3 \pi^{2}} \int_{0}^{\infty} d k k^{2} g_{k}^{2}$ and $c_{2}=\frac{m^{2}}{3 \pi^{2}} \int_{0}^{\infty} d k g_{k}^{2}$ quasimolecular size (UV cutoff) dependent constants.

Firstly, we observe that for a rotationally-invariant case of $\epsilon_{\alpha}=\epsilon_{0}$, Eq. (2) reduces to the $\ell=1(p$ wave) case of the most general expression for the scattering amplitude, $f_{\ell}(p)=\left[p^{-2 \ell} F_{\ell}\left(p^{2}\right)-i p\right]^{-1}$, for scattering from a central potential in angular momentum channel $\ell$, obtained simply from unitarity and analyticity. $F_{\ell}\left(p^{2}\right)$ is a real function Taylor-expandable in powers of its argument[18], that in our case can be read off from Eq. (2) and given exactly (for $\epsilon_{\Lambda} \gg E$ ) by $F_{1}\left(p^{2}\right)=-A+B p^{2}$, where $A=-\left(\epsilon_{0}-c_{1}\right) \frac{6 \pi}{m g_{0}^{2}}$ is the inverse scattering volume, and $B=-\frac{6 \pi}{m^{2} g_{0}^{2}}\left(1+c_{2}\right)$.

Hence, $H$ in Eq. (1) correctly reproduces the lowenergy two-body physics, regardless of the details of real interatomic interactions. The physical detuning position of the FR (corresponding to the molecular state that is real/virtual when it is negative/positive) is given by the low-energy poles of the scattering amplitude $f\left(\mathbf{p}, \mathbf{p}^{\prime}\right)$, $\omega_{\alpha} \equiv \frac{\epsilon_{\alpha}-c_{1}}{1+c_{2}}$, expressed in terms of the bare Hamiltonian parameters $\epsilon_{\alpha}$. In contrast to the $s$-wave case, where such renormalization only shifts the position of the FR, here it also leads to a multiplicative renormalization.

We now proceed to the main topic of the Letter, namely to the study of thermodynamics of a fermionic atomic gas at a finite total density $n$, near a $p$-wave FR described by $H$, Eq. (1). It is convenient to work in the grand-canonical ensemble, $H_{\mu}=H-\mu \hat{n}$, with the chemical potential $\mu$ determined by the average total atom density $n=\frac{1}{V} \sum_{\mathbf{p}}\left(\sum_{\alpha} 2\left\langle\hat{b}_{\alpha \mathbf{p}}^{\dagger} \hat{b}_{\alpha \mathbf{p}}\right\rangle+\left\langle\hat{a}_{\mathbf{p}}^{\dagger} \hat{a}_{\mathbf{p}}\right\rangle\right)$.

$H_{\mu}$ cannot be diagonalized exactly. However, as we will show below, at $T=0$ the system is in a paired SF state, characterized by a finite $\left\langle\hat{b}_{\alpha 0}\right\rangle=\sqrt{V} B_{\alpha}$ for any value of detuning, $\omega_{\alpha}$. This can be easily seen by noting that for a large positive detuning $\omega_{\alpha} \gg 2 \epsilon_{F}$, there is a large gap to creating (quasi-)molecules, $\hat{b}_{\alpha}$. Therefore they can be integrated out to lead to an effective BCS model, with an attractive $p$-wave four-Fermi interaction of strength $\lambda_{B C S}=g_{0}^{2} /\left(\omega_{\alpha}-2 \mu\right)$, with virtual molecules mediating the attractive interactions between fermions. The resulting finite Cooper-pair field $\left\langle\hat{a}_{-\mathbf{p}} \hat{a}_{\mathbf{p}}\right\rangle$ in this BCS regime then always induces a finite (albeit exponentially small) $\left\langle\hat{b}_{\alpha 0}\right\rangle$ through a resonant $g_{0}$ coupling. In the opposite BEC regime, $\omega_{\alpha}<2 \epsilon_{F}$, we will show that $\mu$ decreases, tracking closely $\omega_{\alpha} / 2$, and the energy can be lowered by binding a fraction of atoms into Bose-condensed $p$-wave molecules, stabilized by Pauli-blocking.

Hence, to make progress we approximate the molecular field $\hat{b}_{\alpha \mathbf{p}}$ by its condensate value $\left\langle\hat{b}_{\alpha 0}\right\rangle=\sqrt{V} B_{\alpha}$, neglecting bosonic excitations. This reduces $H_{\mu}$ to a quadratic, mean-field BCS form (with $B_{\alpha}$ playing the role of the Hubbard-Stratonovich field, i.e., the gap function), that can be easily diagonalized by a Bogoliubov transformation.

The key point is, that, independent of $\omega_{\alpha}$, fluctuations about this molecular saddle-point approximation are suppressed in powers of a dimensionless coupling

$$
\gamma=\frac{\sqrt{2}}{3 \pi^{2}} g_{0}^{2} \epsilon_{F}^{1 / 2} m^{5 / 2}
$$

proportional to the ratio of the FR width to Fermi energy. $\gamma$ can always be made sufficiently small by simply reducing the density $n=\left(2 m \epsilon_{F}\right)^{3 / 2} /\left(6 \pi^{2}\right)$ [19]. Hence, for $\gamma \ll 1$, total number of excited molecular bosons $\sum_{\alpha, \mathbf{q} \neq 0}\left\langle\hat{b}_{\alpha \mathbf{q}}^{\dagger} \hat{b}_{\alpha \mathbf{q}}\right\rangle$ is small, and above molecular mean- 
field approximation is quantitatively accurate, with corrections $O(\gamma)$. In the BCS regime, this reduces to the standard criterion for the validity of the BCS meanfield corresponding to the gap much smaller than $\epsilon_{F}$, namely Cooper pairs that are large compared to interatomic spacing[20]. However, in contrast to the standard BCS model, the $\gamma \ll 1$ criterion is far less stringent and allows a quantitatively accurate analysis even in the molecular BEC regime, where from the BCS prospective the atoms are strongly coupled with $\mu$ much smaller than the atomic gap. In this regime the limitation of the molecular mean-field theory simply corresponds to a small depletion of the molecular condensate.

Hence for $\gamma \ll 1$, we can accurately compute the free energy $F\left(B_{\alpha}\right)$. As usual, its minimization with respect to the complex order parameter $\mathbf{B}, \partial F[\mathbf{B}] / \partial B_{\alpha}^{*}=0$ leads to a BEC-BCS gap equation,

$\left(\epsilon_{\alpha}-2 \mu\right) B_{\alpha}=B_{\beta} \int \frac{d^{3} k}{(2 \pi)^{3}} \frac{g_{k}^{2} k_{\alpha} k_{\beta}}{E_{k}} \tanh \left(\frac{E_{k}}{2 k_{B} T}\right)$,

whose solution, together with the total atom conservation equation

$$
n=2 n_{b}+n_{a}
$$

determines the molecular condensate amplitude, $\mathbf{B}$ and the chemical potential, $\mu$ as a function $\omega_{\alpha}, T$ and $n$. In above, $n_{a}(\mu)=\frac{1}{2} \int \frac{d^{3} k}{(2 \pi)^{3}}\left[1-\frac{\frac{k^{2}}{2 m}-\mu}{E_{k}} \tanh \left(\frac{E_{k}}{2 k_{B} T}\right)\right]$ is the number of open-channel BCS-paired atoms, with $E_{k}=\sqrt{\left(\frac{k^{2}}{2 m}-\mu\right)^{2}+4 g_{k}^{2}|\mathbf{B} \cdot \mathbf{k}|^{2}}$ the Bogoliubov atomic quasi-particle spectrum, and $n_{b}=\sum_{\alpha \mathbf{q}}\left\langle\hat{b}_{\alpha \mathbf{q}}^{\dagger} \hat{b}_{\alpha \mathbf{q}}\right\rangle$ the total number of closed-channel molecules.

The complex vector order parameter $\mathbf{B}$ encodes the three molecular states $\psi_{m}$ with spin projection $m=0, \pm 1$ through the relations $\psi_{0}=B_{z}, \psi_{1}=-\frac{1}{\sqrt{2}}\left(B_{x}+i B_{y}\right)$, $\psi_{-1}=\frac{1}{\sqrt{2}}\left(B_{x}-i B_{y}\right)$ [18]. It can be parameterized in terms of two real vectors

$$
\mathbf{B}=\mathbf{u}+i \mathbf{v},
$$

that by a gauge transformation $\mathbf{B} \rightarrow e^{i \varphi} \mathbf{B}$ can always be conveniently chosen to be orthogonal, with $\mathbf{u} \cdot \mathbf{v}=0$. Consequently, if either one (but not both) of the vectors vanishes (e.g., v), then the projection of the angular momentum on the axis parallel to the other vector $(\mathbf{u})$ is equal to zero. If, on the other hand, $u=v$ (with $u=|\mathbf{u}|$ and $v=|\mathbf{v}|)$, then the projection of the angular momentum on $\mathbf{v} \times \mathbf{u}$ is equal to 1 [18].

Focusing first on the $T=0$ case, by integrating Eq. (4) we find the free energy in this $u, v$ representation

$$
\begin{aligned}
& \frac{F(\mathbf{u}, \mathbf{v})}{1+c_{2}}=\sum_{\alpha}\left(u_{\alpha}^{2}+v_{\alpha}^{2}\right)\left[\omega_{\alpha}-2 \mu+a_{1} \ln \left\{a_{0}(u+v)\right\}\right] \\
& +a_{1} \frac{u^{3}+v^{3}}{u+v}+a_{2}\left[\left(u^{2}+v^{2}\right)^{2}+\frac{1}{2}\left(u^{2}-v^{2}\right)^{2}\right],
\end{aligned}
$$

with $a_{1}=\left(\frac{2}{1+c_{2}} \gamma \sqrt{\mu / \epsilon_{F}}\right) \mu, a_{2}=\left(\frac{8}{5} \frac{c_{2}}{1+c_{2}} \gamma\right) \epsilon_{F} / n$, $a_{0}=e^{5 / 6}\left(\mu / \epsilon_{F}\right)^{1 / 4}(\gamma / 8 n)^{1 / 2}$. As usual, the logarithmic term arises from the Fermi surface $\left(k^{2} / 2 m \sim \mu\right)$ contribution to the integral of Eq. (4). It is only a good estimate for $\mu>0$ when the logarithm itself is large, and vanishes otherwise. On the other hand, the quartic terms $a_{2}\left[\left(\mathbf{B}^{*} \cdot \mathbf{B}\right)^{2}+\frac{1}{2}|\mathbf{B} \cdot \mathbf{B}|^{2}\right]$ in $F$ arise from momenta $k \sim \Lambda$.

Minimization of $F(u, v)$ leads to the phase diagram as a function of detuning $\omega_{\alpha}$, displayed in Fig.1. For $\omega_{0} \gg 2 \epsilon_{F}$ (BCS regime), the molecular density is vanishingly small and atom conservation, Eq. (5), constrains $\mu$ to be close, but slightly below $\epsilon_{F}$. The minimum of $F(u, v)$ is then controlled by the balance of the quadratic and logarithmic terms in $u$ and $v$, with the quartic terms exponentially small. In the isotropic case with molecular spin-independent FR, $\omega_{\alpha}=\omega_{0}, F$ is invariant under simultaneous rotation of $\mathbf{u}$ and $\mathbf{v}$, and exhibits three (plus symmetry related) local extrema: (i) normal state with $u=v=0$, (ii) $p_{x}$-wave superfluid with $u \neq 0, v=0$, and (iii) $p_{x}+i p_{y}$-wave superfluid with $u=v \neq 0$. The absolute minimum of $F$ is given by (iii) with $u=v \approx \frac{1}{2 a_{0} e} e^{-\left(\omega_{0}-2 \epsilon_{F}\right) / a_{1}}$, and the condensation energy ratio $\mathcal{R}=F_{p_{x}+i p_{y}} / F_{p_{x}} \approx e / 2>1$, robustly giving $p_{x}+i p_{y}$ as the ground state in the BCS regime, consistent with the $A_{1}$ superfluid ground state of a fully spin-polarized ${ }^{3} \mathrm{He} .[11,21,22]$.

In the complementary $\omega_{0}<2 \epsilon_{F}$ crossover regime, it becomes favorable to pair-up a finite fraction of atoms into closed-channel Bose-condensed molecules at energy $\omega_{0}$. This forces $\mu$ to track $\omega_{0} / 2$ below $\epsilon_{F}$. Consequently, the condensate density $n_{b} \approx|B|^{2}$ is no longer exponentially small, growing to a finite fraction of the overall density $n$, and as a result the logarithmic term in Eq. (7) becomes negligible. The condensate density $|B|^{2}$ is then primarily determined by the atom number Eq. (5), with the gap equation simply constraining $\mu$ to track $\omega_{0} / 2$ to the accuracy $\mathcal{O}(\gamma)$. In the isotropic case, it is clear from Eq. (7) that although the quadratic $\mathbf{B}^{*} \cdot \mathbf{B}$ and the first quartic $\left(\mathbf{B}^{*} \cdot \mathbf{B}\right)^{2}$ terms are invariant under rotation in the $u-v$ space, the positive coefficient of the $|\mathbf{B} \cdot \mathbf{B}|^{2}=\left(u^{2}-v^{2}\right)^{2}$ quartic term guarantees that $F(u, v)$ is again minimized by $u=v \approx \frac{1}{2} \sqrt{n-n_{a}\left(\omega_{0} / 2\right)}$, corresponding to the time-reversal-breaking $p_{x}+i p_{y}$-wave superfluid ground state. In this idealized isotropic case, the finite $T$ transition from the normal to the $p_{x}+i p_{y}$ superfluid state is described by the complex $\mathrm{O}(3)$ vector model, $F=t \mathbf{B}^{*} \cdot \mathbf{B}+\lambda_{1}\left(\mathbf{B}^{*} \cdot \mathbf{B}\right)^{2}+\lambda_{2}|\mathbf{B} \cdot \mathbf{B}|^{2}$.

However, in the experimental realization, the magnetic dipole interaction generically splits the FR into two $m=0$ and $m= \pm 1$ resonances[9]. Choosing $x$-axis along the external magnetic field, this can be captured in our model Eq. (1) by taking $\omega_{x}=\omega_{0}-\delta \neq \omega_{y}=$ $\omega_{z} \equiv \omega_{0}$. This orbital easy axis (experimentally $\delta>0$ ) anisotropy substantially enriches the phase diagram aris- 
ing from minimization of $F[\mathbf{u}, \mathbf{v}]$, Eq.7. A technically involved analysis [8] predicts for an intermediate FR splitting $1 /\left(1+c_{2}\right)<\delta /\left(n a_{2}\right)<5 /\left(4 c_{2}\right)$ (Fig. 1(a)), that for $\omega_{0}<\omega_{0 c}$ the free-energy is minimized by a $\mathbf{u}=u \hat{x}$, $\mathbf{v}=0$ state corresponding to a $p_{x}$-wave $m=0$ superfluid. At low $T$, for $\omega_{0}>\omega_{0 c}$, we instead find that the free-energy is minimized by a $u \neq v \neq 0$, characterizing a state that breaks time-reversal symmetry and is a coherent superposition of $p_{x}$ and $p_{x}+i p_{y}$ superfluids (that we refer to as simply a $p_{x}+i p_{y}$ state). It is easy to see that the quantum phase transition from the $p_{x}$ to the $p_{x}+i p_{y}$ superfluid is second-order. The global (gauge) $\mathrm{U}(1)$ symmetry is spontaneously broken in the $p_{x}$ superfluid, and rotational $\mathrm{O}(3)$ symmetry is explicitly broken (down to $\mathrm{O}(2)$ ) by the dipole interaction that acts like Ising (easy axis) anisotropy. Hence it can be shown [8] that the $T=0 p_{x}$ to $p_{x}+i p_{y}$ transition at $\omega_{0 c}$ is in the $4 \mathrm{D}$ $\mathrm{O}(2)$ (XY) universality class. The corresponding critical point controls the shape of the low $T$ phase boundary with $T_{c 1}\left(\omega_{0}\right) \sim\left|\omega_{0}-\omega_{0 c}\right|^{z \nu}$, and $z=1$ and $\nu=\frac{1}{2}$.

From the general structure of $F$ near $T_{c 2}\left(\sim \epsilon_{F}\right.$ for $\omega_{0} \lesssim 2 \epsilon_{F}$ and exponentially small in the BCS regime), where the nonanalytic logarithmic term in Eq. (7) is suppressed (through the hyperbolic tangent in Eq. (4)), it is possible to show that for an arbitrary small FR splitting $\delta>0$ the normal-superfluid transition is always into the $p_{x}$ state (with the transition in the 3D XY universality class), in contrast to the isotropic case, $\delta=0$, where it is always into the $p_{x}+i p_{y}$ superfluid[8].

For a sufficiently small FR splitting $\delta /\left(n a_{2}\right)<1 /(1+$ $c_{2}$ ) (Fig. 1(b)), the $T=0$ phase transition disappears and at low $T$ the $p_{x}+i p_{y}$ superfluid extends to all detuning, undergoing a transition to the $p_{x}$ state at $T_{c 1}\left(\omega_{0}\right)$ $\left(\sim \epsilon_{F}\right.$ for $\left.\omega_{0} \lesssim 2 \epsilon_{F}\right)$ only near the normal-to-superfluid $T_{c 2}\left(\omega_{0}\right)$, below which the $p_{x}$ superfluid is confined in a sliver of $T_{c 1}<T<T_{c 2}$ set by $\ln \left[T_{c 2} / T_{c 1}\right] \sim \delta$. This is consistent with the isotropic case $\delta=0$, where $p_{x}+i p_{y}$ state extends all the way up to $T_{c 2}$. In contrast, for a large FR splitting (Fig. 1(c)), $\delta /\left(n a_{2}\right)>5 /\left(4 c_{2}\right)$, it is clear from $F$, Eq. (7) that the dipole anisotropy dominates all other terms and leads to the $p_{x}$ state over the full $T-\omega_{0}$ phase diagram. However, it is important to note that for a very large FR splitting, $\delta$, given long energy relaxation times, near the $m=1$ resonance the atomic gas may remain in a metastable $p_{x}+i p_{y}$ state, despite the existence of a lower energy $p_{x}$ state.

The analysis presented in this Letter establishes the stability of $p_{x}+i p_{y}$ SF phase over a large portion of the phase diagram, in an experimentally realizable system in which the chemical potential $\mu$ can be directly tuned through zero by adjusting FR detuning $\omega_{0}$. This allows us to take advantage of the established literature $[6,14,16,17]$, that demonstrates that such SF state undergoes a topological phase transition at $\mu=0$ between two types of $p_{x}+i p_{y}$ SFs. In 3D these are distinguished by the existence of gapless atomic excita- tions for $\mu>0$ (due to nodal points at $\mathbf{p}= \pm p_{F} \hat{\mathbf{z}}$ ) and a fully gapped spectrum for $\mu<0$. In $2 \mathrm{D}$ (to which our computation of $F$ can be easily extended without any qualitative changes [8]) both of these states are fully gapped but nevertheless are distinguished by topological order $[16,17]$. Thus our work explicitly shows that degenerate Fermi gases near a $p$-wave FR give a concrete realization of non-Abelian topological states, which are of interest in strongly correlated systems with possible applications to fault-tolerant quantum computing[23]. Detecting phases of $p$-wave superfluids and transitions between them (illustrated in Fig. 1) in cold atomic gases remains an open problem.

We acknowledge support by the NSF DMR-0321848 (L.R.), DMR-9984002 (A.A.), and the Packard Foundation (A.A., L.R.), and thank J. Bohn, and D. Jin for discussions.

Note added: After this paper was posted, a manuscript by Chi-Ho Cheng and S.-K. Yip [24] appeared that studied the same problem. For large $\left(\delta /\left(n a_{2}\right)>5 /\left(4 c_{2}\right)\right)$ and small $\left(\delta /\left(n a_{2}\right)<1 /\left(1+c_{2}\right)\right)$ Feshbach resonance splitting their results coincide with ours (summarized by our Figs. $1(\mathrm{~b}, \mathrm{c})$ ). However, results disagreed (see Fig. 1(a) in version 2 of our manuscript) for the narrow range of the intermediate values of the Feshbach resonance splitting, $1 /\left(1+c_{2}\right)<\delta /\left(n a_{2}\right)<5 /\left(4 c_{2}\right)$, for reasons at the time not understood by either groups. Upon further analysis we found that Cheng and Yip's predictions [24] for this narrow range of FR splitting was correct. Our mistake stemmed from not appreciating the dominant (in the narrow intermediate range of FR splitting) contribution to the atom number coming from the long tail in the atomic momentum distribution function. Correction of this mistake is reflected in the updated version of Fig. 1(a), but does not affect any other result in our original manuscript. We credit Cheng and Yip for the correct version of Fig. 1(a). Detailed analysis will be presented in the manuscript currently in preparation [25].

We regret that due to our oversight this note did not make it into the version of our manuscript published in Physical Review Letters.

[1] C. A. Regal et al., Phys. Rev. Lett. 92, 040403 (2004).

[2] M. Bartenstein et al, Phys. Rev. Lett. 92, 120401 (2004).

[3] M. W. Zwierlein et al, Phys. Rev. Lett. 92, 120403 (2004).

[4] E. Timmermans et al., Phys. Lett. A 285, 228 (2001).

[5] M. Holland et al., Phys. Rev. Lett. 87, 120406 (2001).

[6] A. Leggett, in Modern Trends in the Theory of Condensed Matter (Springer-Verlag, Berlin, 1980), pp. 13-27.

[7] P. Nozières and S. Schmitt-Rink, J. Low Temp. Phys. 59, 195 (1985).

[8] V. Gurarie, L. Radzihovsky, and A. V. Andreev, unpublished. 
[9] C. Ticknor et al., Phys. Rev. A 69, 042712 (2004).

[10] C. H. Schunck et al., cond-mat/0407373.

[11] T.-L. Ho and R. B. Diener, Phys. Rev. Lett. 94, 090402 (2005).

[12] Y. Ohashi, Phys. Rev. Lett. 94, 050403 (2005).

[13] S. Botelho and C. A. R. S. de Melo, cond-mat/0409357.

[14] F. R. Klinkhamer and G. E. Volovik, Pisma Zh. Eksp. Teor. Fiz. 80, 389 (2004).

[15] G. Moore and N. Read, Nucl. Phys. B 360, 362 (1991).

[16] G. E. Volovik, Exotic properties of superfluid ${ }^{3} \mathrm{He}$ (World Scientific, Singapore, 1992).

[17] N. Read and D. Green, Phys. Rev. B 61, 10267 (2000).

[18] L. D. Landau and E. M. Lifshitz, Quantum Mechanics (Butterworth-Heinemann, Oxford, UK, 1981).
[19] Contrast this with the $s$-wave BCS-BEC superfluid, where the dimensionless coupling is $m^{\frac{3}{2}} g^{2} / \sqrt{\epsilon_{F}}$, that descreases at (not easily reachable) high density.

[20] This mean-field approximation remains valid even in the regime $\epsilon_{\Lambda} \gg \epsilon_{F}$ of interest to us, a limit that has not been considered in condensed matter systems.

[21] D. Vollhardt and P. Wölfle, The Superfluid Phases of $\mathrm{He}^{3}$ (Taylor \& Francis, N.Y., 1990).

[22] P. W. Anderson and P. Morel, Phys. Rev. 123, 1911 (1961).

[23] A. Kitaev, Ann. Phys. 303, 2 (2003).

[24] C.-H. Cheng and S.-K. Yip, cond-mat/0504278.

[25] V. Gurarie and L. Radzihovsky, in preparation (2005). 